\title{
Information Design in Coordination Games with Risk Dominant Equilibrium Selection'
}

\section{By: Michael Ebert, Joseph Kadane, Dirk Simons and Jack Stecher}

\begin{abstract}
We study the design of public information structures that maximize the probability of selecting a Pareto dominant equilibrium in symmetric $(2 \times 2)$ coordination games. Because the need to coordi-nate exposes players to strategic risk, we treat the designer as able to implement an equilibrium only if the players believe it is also risk dominant. The designer's task is therefore to pool the set of states in which the desired equilibrium is risk dominant with the largest possible set in which it is not, while keeping the desired equilibrium risk dominant in expectation. We provide a simple characterization of the optimal signal structure which holds under general conditions. We extend the analysis to related problems, and show that our intu-ition is robust, suggesting that our approach provides a promising way forward for a large class of problems in constrained information design.
\end{abstract}

Keywords: Bayesian persuasion, coordination, information design, Neyman-Pearson lemma, risk dominance. 


\section{Introduction}

Information is useful for coordination, because it gives players a way to correlate their strategies. But it is well known that information can also sabotage coordination, if it leads players to select an undesirable equilibrium. Common examples are bank runs and investor panics (Kaplan, 2006; Qu, 2013; Vives, 2014; de Oliveira Cavalcanti and Monteiro, 2016), self-fulfilling sovereign debt crises (Cole and Kehoe, 2000), and currency attacks (Morris and Shin, 1998). We are interested in characterizing information structures that maximize the chance of selecting a desirable equilibrium in common interest coordination games of imperfect information.

Our focus on common-interest games ensures that the equilibria are Pareto ranked, so that there is universal agreement on which equilibrium is desirable. However, this unanimity does not determine which equilibrium the players select. Rather than basing their strategies solely on Pareto dominance, players might also consider how sensitive their payoffs are to other players' decisions. A common selection criterion that incorporates strategic risk is risk dominance (Harsanyi and Selten, 1988), which we describe in detail below. This criterion has solid theoretical foundations (Crawford, 1991; Carlsson and van Damme, 1993; Morris and Shin, 2002, 2003) and is on balance supported experimentally (van Huyck et al., 1990; Schmidt et al., 2003; Cabrales et al., 2007; Anctil et al., 2010). ${ }^{1}$

Whether the Pareto dominant equilibrium is also risk dominant depends on the expected payoffs. Changing the information available to the players, therefore, can modify the ex ante probability that the Pareto dominant equilibrium is also risk dominant, and hence likely to be selected.

Thus, we consider the problem of a social planner, called a designer, who is interested in implementing the Pareto dominant equilibrium and who decides ex ante about the information that players will receive about their

\footnotetext{
${ }^{1}$ Even in experiments that do not fully support risk dominance, such as Heinemann et al. (2009), participant decisions appear to focus on strategic risk.
} 
payoffs. The information the players receive implicitly comes with the designer's recommendation of which equilibrium to play.

This problem differs from the standard information design problem, in which the players are willing to implement any equilibrium the designer recommends. In a setting with Pareto ranked equilibria, the designer in a standard problem simply suggests the Pareto dominant equilibrium, and the players comply, so the designer is unconstrained. At the opposite end of the spectrum, Goldstein and Huang (2016), Inostroza and Pavan (2020), and Mathevet et al. (2020) consider an information designer who faces adversarial players, who choose the equilibrium the designer likes least. In their setting, the designer is maximally constrained. It is easy, however, to imagine situations in which the players are neither perfectly obedient nor uncompromisingly rebellious. The designer chooses an information structure to maximize the probability that her recommended equilibrium is Pareto dominant, subject to the constraint that the recommendation must also be risk dominant.

We find that there is an elegant, natural solution to the designer's problem: she pools the states on which the Pareto dominant equilibrium is also risk dominant with those states on which the Pareto dominant equilibrium is not risk dominant, but close. In particular, she finds a constant tolerance level, and pools all states in which the Pareto dominant equilibrium does not violate risk dominance by more than this tolerance. She chooses the tolerance level so that, conditional on her recommending the Pareto dominant equilibrium, the players know it is weakly risk dominant in expectation.

To elaborate, consider a symmetric common interest game with two equilibria. Let $z(\omega)$ be the state-contingent payoff to each player in the Pareto dominant equilibrium, and let $x(\omega)$ be the corresponding payoff in the alternative equilibrium. Suppose that, for some $\alpha>1$, the Pareto dominant equilibrium is also risk dominant if and only if $z(\omega)$ is at least $\alpha x$. We show that the solution to the designer's problem is to reveal whether $z(\omega) \geq \alpha x(\omega)-k$ 
for some constant $k>0$ that does not depend on $\omega$. If this inequality holds, the designer recommends the Pareto dominant equilibrium. In this case, either the Pareto dominant equilibrium is risk dominant, or, if not, perturbing $z$ by adding at most $k$ to it would make it so. The designer chooses $k$ to be as large as possible, but must keep the expectation of $z$ at least $\alpha x$, conditional on the recommendation to play the Pareto dominant equilibrium. The optimal tolerance level $k$ depends on the prior distribution of states, but the fact that the tolerance is a constant does not.

Having established this result, we turn our attention to several extensions. For example, we consider robustness to changing the designer's objective. Instead of maximizing the probability of implementing the Pareto dominant equilibrium, a designer might be concerned with maximizing the players' expected payoffs. This objective would be natural in the case where one of the players is assigned the role of the designer.

As we show, this modified problem also has a simple characterization: the designer optimally chooses a scaling factor, rather than a constant tolerance. If the designer recommends the Pareto dominant equilibrium, the players know that the recommendation is either risk dominant or, if not, at most some fixed percentage away.

The ways in which we establish these results are similar, and would apply equally well to a large collection of objectives. The method for finding the optimal information design has natural interpretations in hypothesis testing and consumer theory, giving a remarkably easy and intuitive approach to constrained information design.

The structure of the rest of this paper is as follows. Section 2 presents our model. Section 3 shows a simple example that illustrates the solution to the designer's problem. Section 4 gives the main results. Section 5 addresses robustness issues. Section 6 concludes. Proofs are in an appendix. 


\section{The Model}

There are three actors in the model, a designer (she) and two players (both he) of a symmetric $(2 \times 2)$ game of imperfect information. Label the players as $\{1,2\}$. All parties have rational expectations.

Players move simultaneously. Each player's action set is $\{L, R\}$. Let $s_{i}$ be the action of player $i \in\{1,2\}$.

The payoffs in the game depend on an unknown state. Let $(\Omega, \mathcal{F}, \psi)$ be a probability space with a common prior $\psi$, and let

$$
\Omega \underset{\ell}{\stackrel{r}{\rightleftarrows}} \mathbb{R}_{+}
$$

be two $\mathcal{F}$-measurable random variables. Where no confusion can arise, we write $\psi(\omega)$ rather than $\psi(\{\omega\})$.

Given the state $\omega \in \Omega$ and the action profile $\left(s_{1}, s_{2}\right) \in\{L, R\}^{2}$, the payoff to player $i$ is

$$
u_{i}\left(s_{i}, s_{j} ; \omega\right)= \begin{cases}\ell(\omega), & \text { if } s_{i}=L \\ r(\omega), & \text { if } s_{i}=s_{j}=R \\ 0, & \text { if } s_{i}=R, s_{j}=L\end{cases}
$$

Assume that $r(\cdot)$ weakly statewise dominates $\ell(\cdot)$. As we show below, this assumption is not strictly necessary, and we make it purely for convenience.

The players are expected utility maximizers who make their decisions at an interim stage, after observing a public signal but before learning the state. There are two pure strategy equilibria, $(R, R)$ and $(L, L)$, in which $(R, R)$ is Pareto dominant, but choosing $R$ involves strategic risk. ${ }^{2}$ See Figure 1.

The strategic risk may induce the players to coordinate on the Pareto dominated equilibrium $(L, L)$. The examples in Figure 2 illustrate. In both examples, the $(R, R)$ equilibrium is Pareto dominant, giving each player a

\footnotetext{
${ }^{2}$ There is a mixed strategy equilibrium, but it is uninteresting. It is never risk dominant, and it is always Pareto-dominated, so we do not mention it further.
} 
Player 2

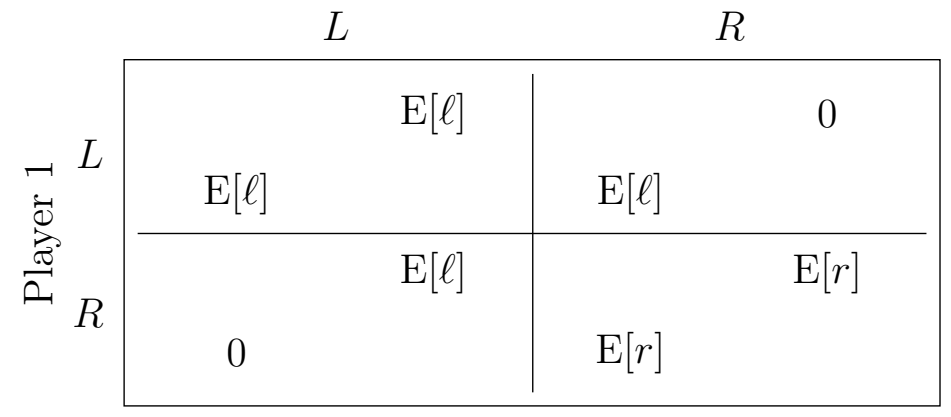

Figure 1: Payoff matrix of the $(2 \times 2)$ game

payoff of 10. In the example on the left, each player receives 9 in the $(L, L)$ equilibrium; in the one on the right, each player receives 1 in $(L, L)$.
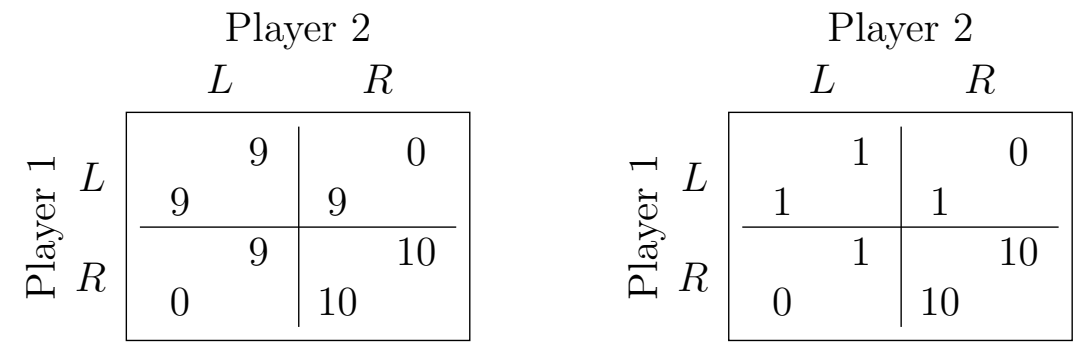

Figure 2: Example payoffs

Each player evaluates strategic risk in terms of the losses he would incur from playing a strategy that does not match the equilibrium the other player tries to select, known as the player's deviation loss. In the example on the left of Figure 2, a player who deviates from the $(R, R)$ equilibrium by choosing $L$ receives 9 utils instead of 10, so his deviation loss is 1 . In the example on the right, deviating from $(R, R)$ to play $L$ means that the player receives 1 instead of 10, a deviation loss of 9. Harsanyi and Selten (1988) define an equilibrium as (weakly) risk dominant if the product of the deviation losses is (weakly) greater than the product of the deviation losses of any other equilibrium. The idea is that the risk of deviating from an equilibrium increases in the 
amount the players stand to lose from deviating. In the example on the left of Figure 2, the $(L, L)$ equilibrium is risk dominant but Pareto dominated. In the example on the right, the $(R, R)$ equilibrium is both Pareto dominant and risk dominant.

We assume that the players are willing to select an equilibrium only if it is weakly risk dominant. In our setting, the symmetry of the game makes the risk dominance criterion simple. From Figure 1, the loss in expected utility to each player from deviating from the $(R, R)$ equilibrium is $\mathrm{E}[r]-\mathrm{E}[\ell]$. Similarly, the expected utility loss to each player from deviating from the $(L, L)$ equilibrium is $\mathrm{E}[\ell]$. Because the game is symmetric, the players face the same deviation losses, so the Pareto-dominant equilibrium is weakly risk dominant if and only if

$$
\mathrm{E}[r] \geq 2 \mathrm{E}[\ell]
$$

The designer's objective is to maximize the probability that the players select the Pareto-dominant equilibrium. Her payoff is

$$
u_{D}\left(s_{1}, s_{2}\right)= \begin{cases}1, & \text { if } s_{1}=s_{2}=R \\ 0, & \text { otherwise }\end{cases}
$$

This is standard in the literature on information design and on Bayesian persuasion (see Kamenica and Gentzkow, 2011; Kolotilin et al., 2017; Bergemann and Morris, 2019; Taneva, 2019; Inostroza and Pavan, 2020; Mathevet et al., 2020). Intuitively, the designer cares only about Pareto efficiency. Without the assumption that $r$ statewise dominates $\ell$, (3) would not correspond to an intrinsic preference for Pareto optima, but instead would reflect a private gain from the $(R, R)$ equilibrium (for example, see the accounting and corporate finance settings of Göx and Wagenhofer, 2009; Carvajal et al., 2018). We discuss this and alternative objectives in Section 5.

Before the players make their decisions and before the state is known, the designer partitions $\Omega$ into three subsets, say $G_{0}, G_{1}$, and $B$. As Bergemann 
and Morris (2019) point out, it suffices for the designer to limit herself to two possible messages, say $g$ or $b$. She will recommend one of two equilibrium strategy profiles, either $(R, R)$ or $(L, L)$, so her message needs to convey only which equilibrium she advises the players to select. She sends message $g$ for sure if $\omega \in G_{0}$, optimally randomizes between $g$ and $b$ if $\omega \in G_{1}$, and sends message $b$ if $\omega \in B$. She can confine attention to public signals due to the strategic complementarities and symmetric information; see Bergemann and Morris $(2019$, p. 60). We refer to $\{g, b\}$ as her signal structure.

The players observe the public signal $g$ or $b$ and then make their decisions. The partition and the designer's recommended strategy are common knowledge. We summarize the sequence of events in Figure 3.

\begin{tabular}{cccc}
\hline & $\mid$ & & \\
\hline designer & nature & public & players \\
chooses & draws & signal $g$ or & choose \\
$\left(G_{0}, G_{1}, B\right)$ & $\omega \in \Omega$ & $b$ revealed & strategies; \\
& & & payoffs are \\
& & & realized
\end{tabular}

Figure 3: Timeline

\section{Illustration}

To see how the designer solves her problem, consider the three-state example shown in Table 1.

Table 1: Payoffs and probabilities in the three-state example

\begin{tabular}{lccc} 
& $\omega_{1}$ & $\omega_{2}$ & $\omega_{3}$ \\
\hline$r\left(\omega_{i}\right)$ & 9 & 4 & 3 \\
$\ell\left(\omega_{i}\right)$ & 5.5 & 0 & 2 \\
\hline $\operatorname{Prob}\left(\omega_{i}\right)=\psi_{i}$ & 0.5 & 0.2 & 0.3 \\
\hline
\end{tabular}


In the equilibrium $(R, R)$, each player receives 9 in state $\omega_{1}, 4$ in $\omega_{2}$, and 3 in $\omega_{3}$. Without any further information, the expected payoff to each player in the $(R, R)$ equilibrium is 6.2 . In the $(L, L)$ equilibrium, the payoffs are 5.5 in $\omega_{1}, 0$ in $\omega_{2}$, and 2 in $\omega_{3}$, or 3.35 in expectation. Since $6.2<2 \cdot 3.35=6.7$, the $(L, L)$ equilibrium is strictly risk dominant.

If the designer provides no information on the state, the players would, therefore, select the Pareto dominated equilibrium $(L, L)$. An easy improvement would be to disclose the state. Equivalently, she could let $G_{0}=$ $\left\{\omega_{2}\right\}, G_{1}=\varnothing$, and $B=\left\{\omega_{1}, \omega_{3}\right\}$, telling the players whether, conditional on the state, $(R, R)$ is risk dominant. Coordination is successful with probability 0.2 , and each player's expected payoff increases by $4 \cdot 0.2=0.8$. This design strategy is suboptimal, but a step in the right direction.

To find the designer's optimal strategy, we imagine her as a consumer, who wants to obtain as much of $\Omega$ as she can. The amount of $\Omega$ she consumes corresponds to the set of states in $G_{0}$ plus her randomization probability weighted set of states in $G_{1}$. In this way, we equate her consumption with the probability of recommending $(R, R)$.

If the designer can access an external randomization device, she could include state $\omega$ in her consumption set with a probability $q$ between 0 and $\psi(\omega)$. Viewing the designer as a consumer, we imagine that $\psi(\omega)$ is the amount of $\omega$ available to her. The quantity $q$ she purchases of $\omega$ is restricted to $[0, \psi(\omega)]$.

The cost to the consumer of $q$ units of the state- $\omega$ commodity is $[2 \ell(\omega)-$ $r(\omega)] \cdot q$, the amount that consuming $q$ moves the expected payoff conditional on $g$ toward or below the risk dominance constraint. So we think of $p(\omega):=2 \ell(\omega)-r(\omega)$ as the price of the state- $\omega$ commodity. Some states may have a negative price, such as $\omega_{2}$ in the example. These states constitute her endowment because $(R, R)$ is risk dominant. Therefore, the consumer's 
problem in an $n$-state world is

$$
\max _{q_{1}, \ldots, q_{n}} U\left(q_{1}, \ldots, q_{n}\right)=\sum_{i=1}^{n} q_{i}
$$

subject to

$$
\begin{array}{rr}
\sum_{i=1}^{n} p_{i} q_{i} \leq 0 & \text { (budget constraint) } \\
(\forall i \in\{1, \ldots, n\}) q_{i} \leq \psi_{i} & \text { (capacity) } \\
(\forall i \in\{1, \ldots, n\}) q_{i} \geq 0 & \text { (nonnegativity) }
\end{array}
$$

The consumer's marginal utility of $q_{i}$ is constant and equal across all $i \in$ $\{1, \ldots, n\}$, because she does not care how she increases the probability of reporting $g$, only that the overall probability of coordinating on the Pareto dominant equilibrium is as large as possible. We discuss other objectives below.

We solve the consumer's problem in steps, using the familiar approach from linear programming. First, she consumes to full capacity all states with negative prices. In the three-state example of Table $1, p_{2}=2 \ell\left(\omega_{2}\right)-$ $r\left(\omega_{2}\right)=-4$, so she consumes $q_{2}^{*}=\psi_{2}=0.2$. This creates slack in her budget constraint (5), which we state above in the form of a self-financing constraint. Similarly, if there are states with a zero price, she would consume those to capacity.

Next, among the states with positive prices, she considers the ratio of the marginal utility of each $q_{i}$ to its price. In the example,

$$
\frac{M U_{1}}{p_{1}}=\frac{1}{2}<\frac{1}{1}=\frac{M U_{3}}{p_{3}}
$$

Therefore, she always gets more marginal utility from a marginal expenditure on $q_{3}$ than from a marginal expenditure on $q_{1}$. She consumes $q_{3}$ until either 
her capacity constraint or her budget constraint binds. If she consumes $q_{3}$ to full capacity, her self-financing condition (5) is

$$
p_{2} \psi_{2}+p_{3} \psi_{3}=-4 \cdot 0.2+1 \cdot 0.3=-0.5<0
$$

so she has resources left in her budget. The only commodity left is $q_{1}$, and as it has positive marginal utility, she consumes it until she exhausts either its capacity or her budget.

We have already seen that she cannot exhaust capacity on $q_{1}$. Doing so would make $G_{0}=\Omega$, i.e., the designer would provide no information. However, we saw above that, with no information, the $(L, L)$ equilibrium is risk dominant. Instead, she spends the rest of her budget on $q_{1}$. From (5) and (8),

$$
p_{1} q_{1}^{*}-0.5=0
$$

so that

$$
q_{1}^{*}=\frac{0.5}{p_{1}}=0.25
$$

The designer's optimal information system works as follows: the designer flips a fair coin. If the coin lands Tails and the state is $\omega_{1}$, she reports $b$. In all other cases, she reports $g$. In this way, she purchases half of the 0.5 probability mass in state $\omega_{1}$ and all of the probability mass in states $\left\{\omega_{2}, \omega_{3}\right\}$. The maximized probability of coordination on $(R, R)$ is 0.75 , compared with the 0.2 she would achieve by fully disclosing the state. The expected payoff to each player increases from 4.15 if she fully discloses the state to 5.325 .

Stepping back, we can interpret the solution to the designer's problem in terms of a linear boundary. She includes in $G_{0}$ any state $\omega$ with $r(\omega)>$ $2 \ell(\omega)-2$, in this case, states $\omega_{2}$ and $\omega_{3}$. For state $\omega_{1}$, she has exactly $r(\omega)=2 \ell(\omega)-2$. In this state, she randomizes, i.e., $\omega_{1} \in G_{1}$. The intercept of -2 is specific to the payoffs and the distribution of states, but as we show in the next section, the slope of 2 is generic.

In sum, the designer solves her problem of constrained information design 
using familiar tools from introductory micro. She views each state $\omega$ as a commodity, and ranks any two states by comparing their marginal utility to price ratios. Therefore, there are two possibilities. If each state has a different marginal utility to price ratio, the designer randomizes on at most one state, consuming all others either to their full capacity or not at all, as in this example. Otherwise, any two states on the boundary must have the same marginal utility to price ratio. This elementary intuition is the key to the general problem.

\section{Results and Interpretation}

Return now to our general setting. The designer's objective is to choose a signal structure $\{g, b\}$ to maximize the probability that, conditional on receiving signal $g$, the $(R, R)$ equilibrium is weakly risk dominant in expectation. Theorem 1 gives the solution.

Theorem 1. The designer maximizes the probability of coordination on the risk dominant equilibrium $(R, R)$ by choosing $g$ as follows: If $\mathrm{E}[r] \geq 2 \mathrm{E}[\ell]$, then $G_{0}=\Omega$ and she always reports $g$. If $\psi(\{\omega \in \Omega \mid r(\omega)>2 \ell(\omega)\})=0$, then $G_{0}=\{\omega \in \Omega \mid r(\omega) \geq 2 \ell(\omega)\}$ and $G_{1}=\varnothing$.

Otherwise, let $K=\{y \in \mathbb{R} \mid \mathrm{E}[r-2 \ell \mid r-2 \ell \geq-y] \geq 0\}$, and let $k=\sup K$. Let $G_{0}=\{\omega \in \Omega \mid r(\omega)>2 \ell(\omega)-k\}$, and let $G_{1}=\{\omega \in \Omega \mid r(\omega)=2 \ell(\omega)-k\}$.

If $k \in K$, the designer's randomization probability is degenerate, i.e., she reports $g$ with probability 1 on $G_{1}$. Otherwise, she reports $g$ with some probability $\gamma<1$ on $G_{1}$, so that $\mathrm{E}[r-2 \ell \mid g]=0$.

The fact that the boundary $r=2 \ell-k$ is affine with a slope of 2 is independent of the prior $\psi$. Only the intercept $-k$ depends on $\psi$.

Remark 4.1. The proof of Theorem 1 uses the fact that the designer's problem is to choose a set of maximal measure subject to a constraint; the solution applies the Neyman-Pearson Lemma. The relationship between the Neyman- 
Pearson Lemma and consumer theory, along with the extensions to allow for randomizing, are discussed in Kadane (1968, p. 160).

Once we see that the key to the designer's problem is the familiar argument of comparing marginal utility to price ratios, the solution is easy to find. As in Section 3, the price of reporting $g$ in state $\omega$ is the amount that doing so reduces $\mathrm{E}[r-2 \ell \mid g]$. Because the designer receives constant marginal utility from any increase in the probability of reporting $g$, the solution to her problem has the simple boundary condition

$$
\frac{\partial U / \partial \omega}{p(\omega)}=\frac{\partial U / \partial \omega^{\prime}}{p\left(\omega^{\prime}\right)} \Leftrightarrow \frac{1}{2 r(\omega)-\ell(\omega)}=\frac{1}{2 \ell\left(\omega^{\prime}\right)-r\left(\omega^{\prime}\right)}
$$

Therefore, if $\omega, \omega^{\prime} \in G_{1}$, it must be the case that

$$
\frac{r(\omega)-r\left(\omega^{\prime}\right)}{\ell(\omega)-\ell\left(\omega^{\prime}\right)}=2
$$

that is, $r$ is affine in $\ell$ with a slope of 2 . Figure 4 illustrates.

In the figure, the support of $(r, \ell)$ is $[a, b]^{2}$. Because $r$ statewise dominates $\ell$, the set of possible ex post values of $r$ and $\ell$ is the right triangle with the $45^{\circ}$-line as its hypotenuse. The set of states in which $(R, R)$ is risk dominant is shown as a dotted right triangle, on and above the $r=2 \ell$ line. This region combined with the interior of the dark gray trapezoid bounded on the right by the $r=2 \ell-k$ line defines the set $G_{0}$. The $r=2 \ell-k$ line defines $G_{1}$. The designer chooses the intercept $(0,-k)$ so that $(\mathrm{E}[\ell \mid g], \mathrm{E}[r \mid g])$ lies on the $r=2 \ell$ line. The light gray irregular quadrilateral defines the set $B$.

It is straightforward to evaluate other constrained information design problems using the same approach. In the next section, we do just that, considering modifications to the problem and to the designer's objective. 


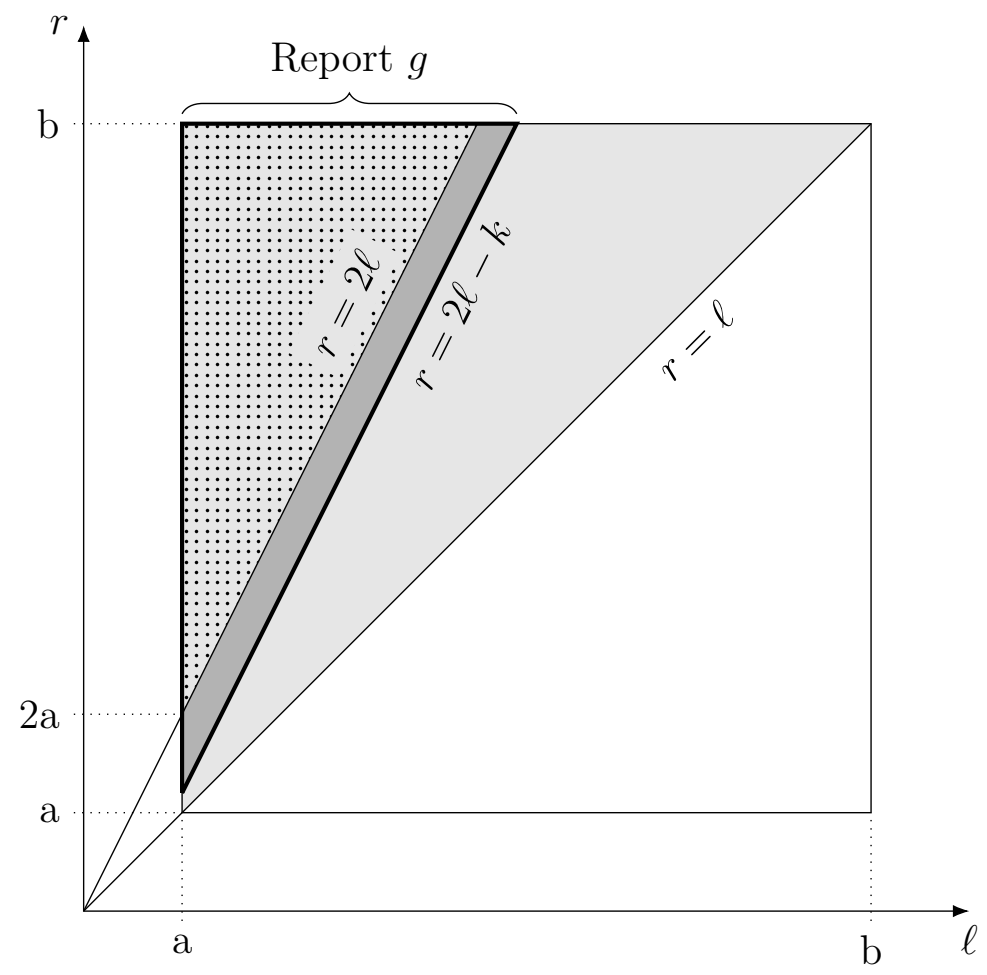

Figure 4: The designer's optimal disclosure region shifts the weak risk dominance line $r=2 \ell$ rightward to $r=2 \ell-k$ with an $r$-intercept $(0,-k)$.

\section{Extensions}

To develop more general intuition on constrained information design, we modify our problem in several ways. We begin by generalizing the players' payoffs (1). Next, we relax the assumption that $r$ statewise dominates $\ell$. Lastly, we alter the designer's objective. 


\subsection{Extension 1: Modified payoffs to the players}

To start, consider the following generalization of the players' payoffs:

$$
u_{i}\left(s_{i}, s_{j} ; \omega\right)= \begin{cases}\ell(\omega), & \text { if } s_{i}=s_{j}=L \\ r(\omega), & \text { if } s_{i}=s_{j}=R \\ \varepsilon(\omega), & \text { if } s_{i}=R, s_{j}=L \\ \ell(\omega)+\delta(\omega), & \text { if } s_{i}=L, s_{j}=R\end{cases}
$$

Assume that in each $\omega \in \Omega, 0 \leq \varepsilon(\omega)<\ell(\omega)<r(\omega)$ and $\delta(\omega)<r(\omega)-\ell(\omega)$. Then $(L, L)$ and $(R, R)$ are equilibria and $(R, R)$ is Pareto dominant.

Proposition 1 says that if $\delta$ and $\varepsilon$ are affine functions of $\ell$ and $r$, the weak risk dominance line is still given by $r$ as an affine function of $\ell$.

Proposition 1. For some constants $c_{1}, c_{2}, c_{3}, d_{1}, d_{2}, d_{3} \in \mathbb{R}$, suppose $\delta(\omega)=$ $c_{1} \ell(\omega)+c_{2} r(\omega)+c_{3}$, and $\varepsilon(\omega)=d_{1} \ell(\omega)+d_{2} r(\omega)+d_{3}$, and that $c_{2}-d_{2}<1$. Then $(R, R)$ is weakly risk dominant if and only if

$$
r(\omega) \geq \frac{2+c_{1}-d_{1}}{1-c_{2}+d_{2}} \ell(\omega)+\frac{c_{3}-d_{3}}{1-c_{2}+d_{2}}
$$

In particular, the weak risk dominance line is affine, and if $\delta$ and $\epsilon$ are constant, then the slope of the weak risk dominance line remains 2.

Intuitively, the hypothesis of Proposition 1 says that the off-equilibrium payoffs can include a lump-sum amount, such as what the players can guarantee for themselves, and potentially some scaled amounts of each of the equilibrium payoffs.

A consequence of Proposition 1 is that if the off-equilibrium payoffs are affine in $\ell$ and $r$, then Theorem 1 is essentially unchanged. The boundary again has the same slope the same as the weak risk dominance line. 


\subsection{Extension 2: State-dependent Pareto dominant equi- librium}

We now return to our assumption that the payoffs to the players are as in (1), and instead drop the assumption that $r$ statewise dominates $\ell$.

It is natural to wonder whether the designer would ever report $g$ for any state $\omega$ for which $\ell(\omega)>r(\omega)$. If the designer intrinsically prefers having the players coordinate on a Pareto dominant equilibrium, then she gains nothing from reporting $g$ in state $\omega$. Her preferences would therefore not be represented by (3), because her interest in getting the players to select $r$ is purely instrumental. Her utility would instead be higher if a Pareto dominant equilibrium is selected, regardless of whether it is $(R, R)$ or $(L, L)$.

If, however, the designer intrinsically prefers $(R, R)$ to $(L, L)$, then her utility is as in (3). For instance, a borrower facing a liquidity shock may want her creditors to roll their short-term debts over. She intrinsically prefers to stay in business, even if she is insolvent. Her utility is (3) even though $(R, R)$ may be Pareto dominated in some states.

Indeed, the designer may find it in her interest to report $g$ for some states in which $(R, R)$ is Pareto dominated. However, including such states is expensive, unless $\ell(\omega)$ and $r(\omega)$ are relatively small. See Figure 5 .

Proposition 2 conveys this idea.

Proposition 2. Let $\omega_{1}, \omega_{2} \in \Omega$ be two states in which $r\left(\omega_{1}\right)>\ell\left(\omega_{1}\right)$ and $r\left(\omega_{2}\right)<\ell\left(\omega_{2}\right)$. Let $r_{1}=r\left(\omega_{1}\right), r_{2}=r\left(\omega_{2}\right), \ell_{1}=\ell\left(\omega_{1}\right)$, and $\ell_{2}=\ell\left(\omega_{2}\right)$. The following are necessary for $\omega_{2} \in G_{0} \cup G_{1}$ and $\omega_{1} \in B$ :

1. $2 \ell_{2}-r_{2} \leq 2 \ell_{1}-r_{1}$, and

2. $r_{2}<\ell_{2}<\ell_{1}<r_{1}$.

That is, the payoffs in state $\omega_{2}$ must be small compared with those in $\omega_{1}$.

Strictness of the first inequality in Proposition 2 is not enough for sufficiency, because the designer might report $g$ or $b$ on both $\omega_{1}$ and $\omega_{2}$. However, 


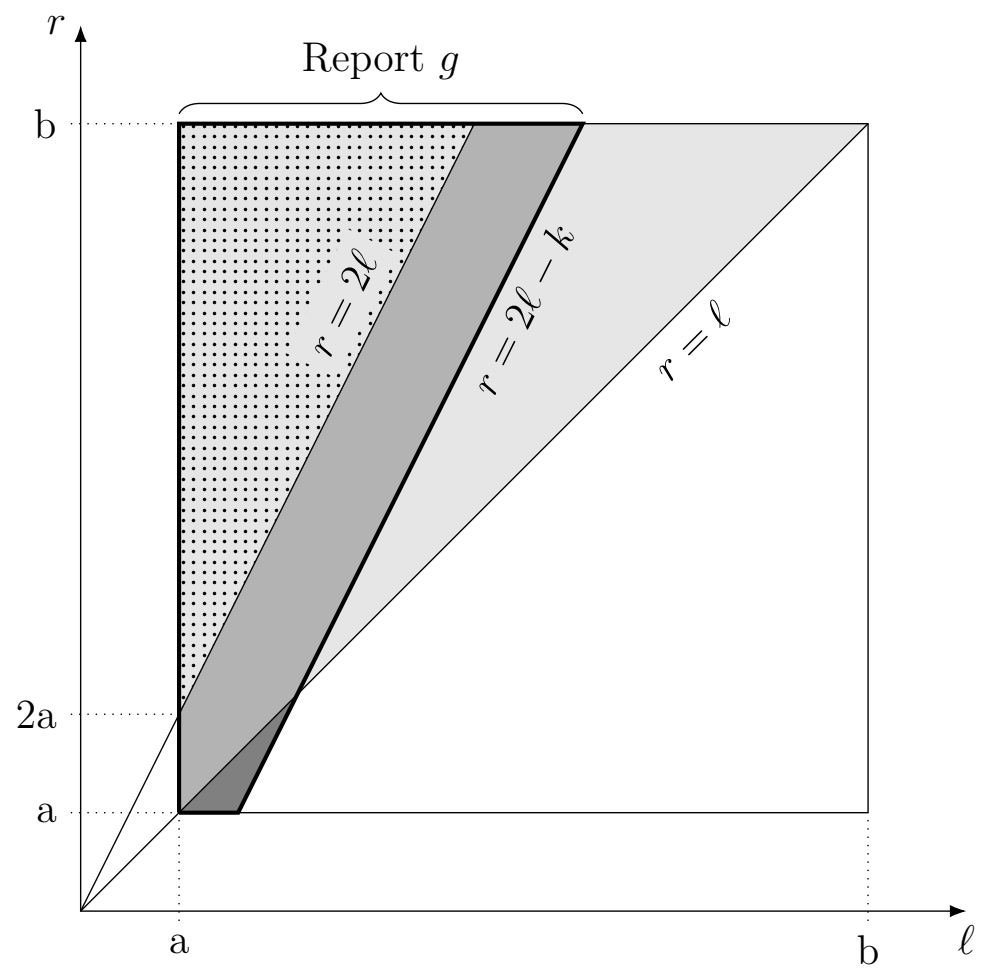

Figure 5: If there is positive probability that $r(\omega)<\ell(\omega)$, the optimal information design is unchanged. The designer might report $g$ in inefficient states where both $r$ and $\ell$ are small (the dark gray obtuse triangle below the $45^{\circ}$ line).

if the first inequality is strict, then $\omega_{1} \in G_{0} \cup G_{1}$ only if $\omega_{2} \in G_{0}$. Nothing changes in the proof of Theorem 1 , so the result still holds.

\subsection{Extension 3: Modified designer objective}

As a final extension, we consider an alternative to the designer's objective. Rather than maximizing the probability that the players select the Pareto dominant equilibrium, the designer might be interested in maximizing the players' expected payoffs. As we shall see, this problem also has an easy 
solution, with the designer's disclosure set as a linear boundary.

To develop some insight, return to the discrete setting of Section 3. The designer's constraints remain (5)-(7). Her objective is now

$$
\max _{q_{1}, \ldots, q_{n}} U\left(q_{1}, \ldots, q_{n}\right)=\sum_{i=1}^{n} q_{i}\left(r_{i}-\ell_{i}\right)
$$

rather than (4). Her marginal utility from adding $q_{i}$ of state $\omega_{i}$ into $G_{0} \cup G_{1}$ is the marginal increment in the players' expected payoffs, $r_{i}-\ell_{i}$.

The designer starts this problem the way our designer in Section 3 does, consuming all states with nonpositive prices to capacity. In the example of Table 1 , she begins by consuming $\omega_{2}$ to its full capacity of $\psi_{2}=0.2$. Her self-financing constraint says that she has spent -0.8 , and therefore does not bind.

As before, she next ranks the states by their marginal utility to price ratio. For arbitrary state $\omega \in \Omega$, this ratio is

$$
\frac{\partial U / \partial \omega}{p(\omega)}=\frac{r(\omega)-\ell(\omega)}{2 \ell(\omega)-r(\omega)}
$$

From the example in Table 1, the designer's marginal utility to price ratio is $4.5 / 2=2.25$ for state $\omega_{1}$ and 1 for state $\omega_{3}$. So she consumes $\omega_{1}$ next. Her self-financing constraint binds if

$$
\begin{aligned}
p_{1} q_{1}+p_{2} q_{2} & =0 \\
\Leftrightarrow 2 q_{1} & =0.8 \Leftrightarrow q_{1}^{*}=0.4
\end{aligned}
$$

This is less than the full capacity of $\psi_{1}=0.5$, so the designer stops here. She sets $G_{0}=\left\{\omega_{2}\right\}$ and $G_{1}=\left\{\omega_{1}\right\}$, setting randomization probability $\gamma^{*}=4 / 5$.

The probability of coordination on the efficient equilibrium is 0.6 in this case, compared with the 0.75 probability that Section 3 shows is attainable. However, the expected payoff is 5.55, compared with 5.325 above. 
For the general case, we observe the boundary ends up as a change in the slope of the weak risk dominance line, rather than a change in the intercept. See Figure 6.

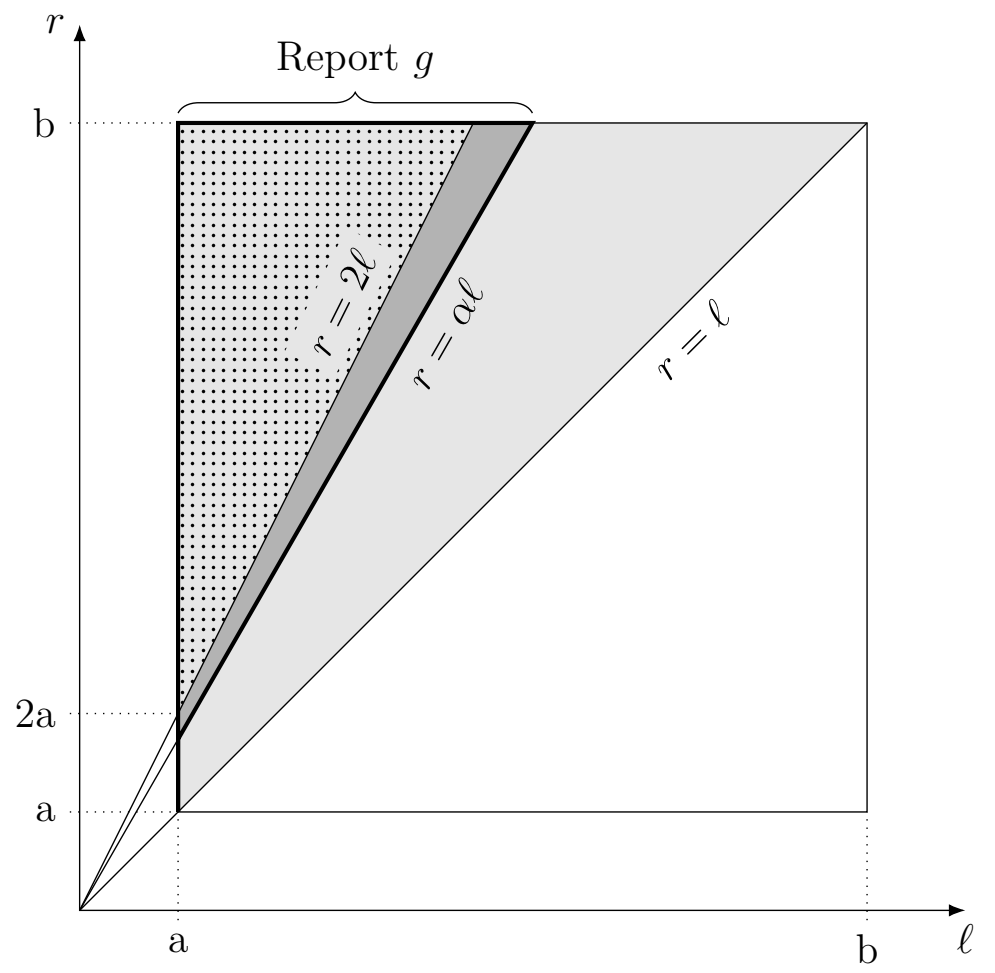

Figure 6: The designer maximizes the expected payoff to the players setting $G_{1}=\{\omega \in \Omega \mid r(\omega)=\alpha \ell(\omega)\}$ for some $\alpha \in(1,2)$.

We state this precisely in Proposition 3. For the proposition, we restrict attention to the case in which $\psi$ is atomless, as extensions to general cases are similar to Theorem 1.

Proposition 3. The designer maximizes the players' expected payoffs as follows: if $\mathrm{E}[r] \geq 2 \mathrm{E}[\ell]$, then set $G_{0}=\Omega$. If $\psi(\{\omega \in \Omega \mid r(\omega)>2 \ell(\omega)\})=0$, then set $G_{0}=\{\omega \in \Omega \mid r(\omega) \geq 2 \ell(\omega)\}$ and $G_{1}=\varnothing$. Otherwise, for some $\alpha \in(1,2)$, set $G_{0}=\{\omega \in \Omega \mid r(\omega)>\alpha \ell(\omega)\}$ and $G_{1}=\{\omega \in \Omega \mid r(\omega)=\alpha \ell(\omega)\}$. 
The slope $\alpha$ is chosen to make $\mathrm{E}[r \mid g]=2 \mathrm{E}[\ell \mid g]$.

In the discrete example, the designer optimally chooses $\alpha=18 / 11$.

\section{Conclusion}

An information designer cannot always take for granted that the receivers of her signal will follow her advice. Our main focus is on a setting in which the players and the designer all prefer the same equilibrium, but may fail to coordinate due to strategic risk.

Our solution has an elegant, linear form, based on intuition from introductory microeconomics. Once we observe that our problem is isomorphic to familiar problems from consumer theory, we are able to tackle other constrained information design problems.

\section{A Proofs}

Proof of Theorem 1. If the prior expectations satisfy $\mathrm{E}[r] \geq 2 \mathrm{E}[\ell]$, then $(R, R)$ is ex ante risk dominant. The designer therefore optimally picks an uninformative signal. Although any babbling equilibrium would be equivalent, we adopt the convention that $G_{0}=\Omega$, i.e., that she announces $g$. At the opposite extreme, if $(R, R)$ is weakly risk dominated with probability 1 , then the designer cannot improve on full transparency, i.e., revealing whether $(R, R)$ is weakly risk dominant for sure.

If neither of these conditions hold, then there is an interior solution to the designer's problem

$$
\begin{aligned}
& \max _{G \in \mathcal{F}} \int_{\omega \in G} d \psi(\omega) \\
& \text { s.t. } \int_{\omega \in G}[2 \ell(\omega)-r(\omega)] d \psi(\omega) \leq 0
\end{aligned}
$$


By the Neyman-Pearson Lemma, the solution has the properties that, for some $c>0$,

$$
\begin{aligned}
& \omega \in G \text { if } 2 \ell(\omega)-r(\omega)<c \\
& \omega \notin G \text { if } 2 \ell(\omega)-r(\omega)>c, \text { and } \\
& \mathrm{E}[r-2 \ell \mid \omega \in G]=0
\end{aligned}
$$

Rearranging, it follows that $G_{0}$ (the set of states that are in $G$ with probability 1$)$ is $\{\omega \in \Omega \mid r(\omega)>2 \ell(\omega)-c\}$ and that $B=\{\omega \in \Omega \mid r(\omega)<2 \ell(\omega)-c\}$. The designer can randomize only on the boundary set $\{\omega \in \Omega \mid r(\omega)=$ $2 \ell(\omega)-c\}$, and by the conclusion of the Neyman-Pearson Lemma, she does so in order to make the risk dominance constraint bind. It remains to show that $c=k$ and that she randomizes if and only if $k \notin K$.

If $c>k$, then $\mathrm{E}[2 \ell-r \mid 2 \ell-r \leq c]>0$, violating the constraint (13). If $c<k$, then either the probability that $2 \ell-r \in(c, k)$ is 0 , in which case we can replace $c$ with $k$, or the designer is not maximizing her objective in (12). It follows that we can always take $c=k$.

If $k \in K$, then $\mathrm{E}[2 \ell-r \mid 2 \ell-r \leq k] \leq 0$, so the designer can report $g$ on all of the boundary $G_{1}$. Otherwise, $\mathrm{E}[2 \ell-r \mid 2 \ell-k \leq k]>0$, so the designer must report $g$ on $k$ with some probability $\gamma$ strictly less than one in order to make the constraint (13) bind.

Proof of Proposition 1. The loss due to deviating from the $(L, L)$ equilibrium is $\ell-\varepsilon$. The loss due to deviating from the $(R, R)$ equilibrium is $r-\ell-\delta$. By the symmetry of the game, the $(R, R)$ equilibrium is weakly risk dominant if 
and only if

$$
\begin{aligned}
r & \geq 2 \ell+\delta-\varepsilon \\
& =2 \ell+c_{1} \ell+c_{2} r+c_{3}-d_{1} \ell-d_{2} r-d_{3} \\
\Rightarrow\left(1-c_{2}+d_{2}\right) r & \geq\left(2+c_{1}-d_{1}\right) \ell+c_{3}-d_{3} \\
r & \geq \frac{2+c_{1}-d_{1}}{1-c_{2}+d_{2}} \ell+\frac{c_{3}-d_{3}}{1-c_{1}+d_{1}}
\end{aligned}
$$

as desired. If $\delta$ and $\varepsilon$ are state-independent, then $c_{1}=d_{1}=c_{2}=d_{2}=0$, giving the slope of 2 .

Proof of Proposition 2. The first condition comes from the associated consumer's problem: each state has the same marginal utility, so if the designer, viewed as a consumer, includes $\omega_{2}$ and does not include $\omega_{1}$, then $\omega_{2}$ must be no more expensive, i.e., $2 \ell_{2}-r_{2} \leq 2 \ell_{1}-r_{1}$.

To get the second condition, rewrite the first one as follows:

$$
\begin{aligned}
2 \ell_{2}-r_{2} & \leq 2 \ell_{1}-r_{1} \\
\Rightarrow \ell_{2}+\left(\ell_{2}-r_{2}\right) & <\ell_{1}+\left(\ell_{1}-r_{1}\right)
\end{aligned}
$$

By hypothesis, the term in parentheses on the left-hand side is positive, and the term in parentheses on the right-hand side is negative. Therefore,

$$
\ell_{2}<\ell_{1}
$$

as desired.

Proof of Proposition 3. As in the proof of Theorem 1, the corner cases of nondisclosure $\left(G_{0}=\Omega\right)$ and full disclosure $\left(G_{0}=\{\omega \in \Omega \mid r(\omega) \geq 2 \ell(\omega)\}\right.$ and $G_{1}=$ $\varnothing)$ are immediate. 
Otherwise, the designer's problem is

$$
\max _{G \in \mathcal{F}} \int_{\omega \in G}[r(\omega)-\ell(\omega)] d \psi(\omega)
$$

subject to (13). We can again apply the Neyman-Pearson Lemma, obtaining for some $d>0$,

$$
\begin{aligned}
& \omega \in G \text { if } \frac{r(\omega)-\ell(\omega)}{2 \ell(\omega)-r(\omega)}<d \\
& \omega \notin G \text { if } \frac{r(\omega)-\ell(\omega)}{2 \ell(\omega)-r(\omega)}>d, \text { and } \\
& \mathrm{E}[r-2 \ell \mid \omega \in G]=0
\end{aligned}
$$

with $d$ chosen to make the constraint bind. Rearranging, the boundary condition for $\omega \in G_{1}$ is

$$
r(\omega)-\ell(\omega)=c[2 \ell(\omega)-2 r(\omega)] \quad \Leftrightarrow \quad r(\omega)-\frac{2 c+1}{c+1} \ell(\omega)
$$

Letting $\alpha=(2 c+1) /(c+1)$ and noting that $c>0$, we see that $1<\alpha<2$.

\section{References}

Anctil, R. M., J. Dickhaut, C. Johnson, And C. Kanodia (2010): "Does Information Transparency Decrease Coordination Failure?" Games and Economic Behavior, 70, 228-241.

Bergemann, D. And S. Morris (2019): "Information Design: A Unified Perspective," Journal of Economic Literature, 57, 44-95.

Cabrales, A., R. Nagel, and R. Armenter (2007): "Equilibrium Selection through Incomplete Information in Coordination Games: an Experimental Study," Experimental Economics, 10, 221-234. 
Carlsson, H. And E. van Damme (1993): "Global Games and Equilibrium Selection," Econometrica, 61, 989-1018.

Carvajal, A., M. Rostek, And G. Sublet (2018): "Information Design and Capital Formation," Journal of Economic Theory, 176, 255-292.

Cole, H. L. And T. J. Kehoe (2000): "Self-Fulfilling Debt Crises," Review of Economic Studies, 67, 91-116.

Crawford, V. P. (1991): "An "Evolutionary" Interpretation of Van Huyck, Battalio, and Beil's Experimental Results on Coordination," Games and Economic Behavior, 3, 25-59.

de Oliveira Cavalcanti, R. And P. K. Monteiro (2016): "Enriching Information to Prevent Bank Runs," Economic Theory, 62, 477-494.

Goldstein, I. And C. Huang (2016): "Bayesian Persuasion in Coordination Games," American Economic Review: Papers and Proceedings, 106, 592-596.

Göx, R. F. And A. Wagenhofer (2009): "Optimal Impairment Rules," Journal of Accounting and Economics, 48, 2-16.

Harsanyi, J. C. And R. Selten (1988): A General Theory of Equilibrium Selection in Games, MIT Press.

Heinemann, F., R. Nagel, and P. Ockenfels (2009): "Measuring Strategic Uncertainty in Coordination Games," Review of Economic Studies, 76, 181-221.

Inostroza, N. And A. Pavan (2020): "Persuasion in Global Games with Application to Stress Testing," Working paper, Northwestern University and University of Toronto.

KadAne, J. B. (1968): "Discrete Search and the Neyman-Pearson Lemma," Journal of Mathematical Analysis and Applications, 22, 156-171. 
Kamenica, E. And M. Gentzkow (2011): "Bayesian Persuasion," American Economic Review, 101, 2590-2615.

Kaplan, T. R. (2006): "Why Banks Should Keep Secrets," Economic Theory, 27, 341-357.

Kolotilin, A., M. Li, T. Mylovanov, and A. Zapechelnyuk (2017): "Persuasion of a Privately Informed Receiver," Econometrica, 85, 19461964.

Mathevet, L., J. Perego, and I. Taneva (2020): "On Information Design in Games," Journal of Political Economy, Forthcoming.

Morris, S. AND H. S. ShIn (1998): "Unique Equilibrium in a Model of Self-Fulfilling Currency Attacks," American Economic Review, 88, 587597.

- (2002): "Social Value of Public Information," American Economic Review, 92, 1521-1534.

- (2003): "Global Games: Theory and Applications," in Advances in Economics and Econometrics, ed. by M. Dewatripont, L. P. Hansen, and S. J. Turnovsky, vol. 1, 56-114.

Qu, H. (2013): "How Do Market Prices and Cheap Talk Affect Coordination?" Journal of Accounting Research, 51, 1221-1260.

Schmidt, D., R. Shupp, J. M. Walker, And E. Ostrom (2003): "Playing Safe in Coordination Games: the Roles of Risk Dominance, Payoff Dominance, and History of Play," Games and Economic Behavior, 42, 281-299.

Taneva, I. (2019): "Information Design," American Economic Journal: Microeconomics, 11, 151-185. 
van Huyck, J. B., R. C. Battalio, and R. O. Beil (1990): "Tacit Coordination Games, Strategic Uncertainty, and Coordination Failure," American Economic Review, 80, 234-248.

Vives, X. (2014): "Strategic Complementarity, Fragility, and Regulation," Review of Financial Studies, 27, 3547-3592. 